Recebido em 12/2017. Aceito para publicação em 01/2020.

\title{
ANÁLISE MICROBIOLÓGICA DE POLPAS DE FRUTAS INDUSTRIALIZADAS COMERCIALIZADAS EM SUPERMERCADO EM BELÉM DO PARÁ
}

\section{MICROBIOLOGICAL ANALYSIS OF INDUSTRIALISED FRUIT PULPS SOLD IN A SUPERMARKET IN BELÉM DO PARÁ}

\author{
Thalita Bandeira Dantas e Silva1 \\ Vanusa de Souza Martins ${ }^{2}$ \\ Juliana Matos Cativo Pereira ${ }^{3}$ \\ Luciane do Socorro Nunes dos Santos Brasil ${ }^{4}$ \\ Suely Maria Ribeiro Da Silva ${ }^{5}$
}

Resumo: As frutas são alimentos de elevado teor nutritivo, compondo uma dieta saudável e equilibrada, possuem ainda um importante papel no desenvolvimento econômico da região Amazônica, que é um bioma com grande diversidade frutífera. Todavia devido as suas características intrínsecas como elevada atividade de água, as frutas tornam-se alimentos altamente perecíveis e com um curto período de comercialização, associado a diversos problemas higiênico-sanitários que envolvem todas as etapas do processamento incluindo a colheita, transporte, armazenamento, beneficiamento e disponibilização para venda. Assim, é fundamental a adoção de adequadas práticas para elaboração das polpas de frutas industrializadas, que se destacam como uma importante alternativa para o aproveitamento dos frutos durante a safra. Visando a segurança microbiológica dos alimentos e saúde de seus consumidores o presente estudo objetivou realizar análises microbiológicas de Coliformes a 45ㄷ e Salmonella spp. de 30 amostras de polpas de frutas comercializados em supermercados de Belém do Pará, com padrão estabelecido pela RDC no12 de 02 de janeiro de 2001. Os resultados obtidos mostraram que 93,34\% das amostras encontravam-se em conformidade em relação a legislação vigente para Coliformes a 45드, além de ausência de Salmonella spp. em 25g, em 100\% das amostras analisadas.

Palavras-chave: Polpa de frutas; análises microbiológicas; coliformes a $45^{\circ} \mathrm{C}$; Salmonella spp.

Abstract: Fruits are foods with high nutritional content that are part of a healthy and balanced diet and play an important role in the economic development of the Amazon region, which is a biome with great fruit diversity. However, because of their intrinsic characteristics such as high water activity, fruits become highly perishable foods with a short period of commercialization, associated with several hygiene and sanitary problems that involve all stages of processing, including harvest, transportation, storage, processing and availability for sale. Therefore, it is fundamental to adopt good practices in the preparation of industrialized fruit pulp, which stand out as an important alternative for the use of fruits during the harvest. Aiming at the microbiological safety of foods and the health of consumers, the present study aimed to perform

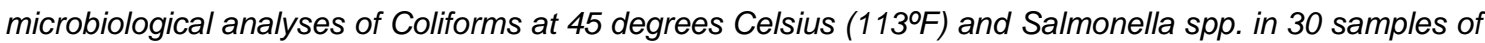
fruit pulps marketed in supermarkets in Belém do Pará, with a standard established by RDC number 12 of January $2^{\text {nd }}, 2001$. The results showed that $93.34 \%$ of the samples were in compliance with the current

\footnotetext{
${ }^{1}$ Doutoranda em Patologia das Doenças Tropicais pela Universidade Federal do Pará - UFPA, Brasil. E-mail: thalitadantas1993@hotmail.com.

2 Graduação em Nutrição - Centro Universitário do Estado do Pará - CESUPA, Brasil. E-mail: vanutri@gmail.com.

${ }^{3}$ Mestre em Oncologia e Ciências Médicas pela Universidade Federal do Pará - UFPA, Brasil. E-mail: juliana_cativo@hotmail.com.

${ }^{4}$ Doutora em Química e Docente e professora adjunto II da Universidade Estadual do Pará - UEPA, Brasil. Email: Isnsbrasil@ig.com.br.

${ }^{5}$ Mestre em Gestão Empresarial pela Universidade Lusófona de Humanidades e Tecnologias (ULHT) e em Ciências e Meio Ambiente pela Universidade Federal do Pará (UFPA); Docente do Centro Universitário do Estado do Pará - CESUPA, Brasil. E-mail: suelynutri@yahoo.com.br.
} 
legislation for Coliforms at $45^{\circ} \mathrm{C}$, in addition to being evidenced the absence of Salmonella spp. in $100 \%$ of the analyzed samples.

Keywords: Fruit pulp; microbiological analyzes; coliforms at $45^{\circ} \mathrm{C}$; Salmonella spp.

\section{INTRODUÇÃO}

A seleção dos alimentos envolve aspectos econômicos, sociais, culturais e valores peculiares de seus consumidores (CRUZ; SCHNEIDER, 2010). Nas diversas áreas do conhecimento, questionamentos que envolvem o tema alimentação e a qualidade são abordados e discutidos de forma ampla, levando a divergências de conceitos e opiniões. $O$ produto adquire conotações positivas e negativas de seus consumidores que influenciarão no momento da compra (HARVEY; McMEEKIN; WARDE, 2004).

A partir da década de 80 , com os intensos avanços tecnológicos e industriais houve uma maior preocupação com os aspectos higiênicos e sanitários que envolviam todas as etapas do processo de produção e comercialização dos alimentos (AQUINO; PHILIPPI, 2002). Um dos grandes desafios no setor alimentício é associar uma produção em escala industrial com a qualidade físico-química e segurança microbiológica do produto (BALBANI; BUTUGAN, 2001).

O controle de qualidade é fundamental em toda cadeia produtiva e comercial, no que se refere a garantia da segurança alimentar (SANTOS; TONDO, 2000). Para que o alimento seja considerado seguro e saudável é necessário considerar uma gama de critérios higiênico-sanitários em todas as etapas de processamento. O aumento do número de casos de doenças transmitidas pelo alimento- DTAs, geralmente está associado a falhas ou inexistência de uma padronização operacional (SIQUEIRA et al, 2010).

Uma das principais fontes de contaminação advém do alimento, que não foi higienizado e/ou não recebeu tratamento térmico adequado. Isto significa que o processamento coerente exerce uma influência positiva na linha produtiva, garantindo a elaboração e venda de um produto seguro, do ponto de vista microbiológico (MESQUITA et al, 2006).

As frutas apresentam diversas características intrínsecas que devem ser cuidadosamente levadas em consideração. Dentre estas, pode-se ressaltar a elevada atividade de água, fato este que contribui para a perecibilidade e reduzido tempo de comercialização do alimento, principalmente no diz respeito a transporte para regiões mais longínquas. Buscando solucionar esse entrave, a indústria alimentícia deve fazer uso de rígidos padrões de qualidade microbiológica e físico-química, evitando danos a saúde de seus consumidores (PARIZ, 2011).

As polpas de frutas necessitam ser submetidas a binômios tempo-temperatura, como é o caso da pasteurização, a fim de que estas possam obedecer a padrões microbiológicos para alimentos especificados na legislação, garantindo assim, a saúde 
dos consumidores. A RDC $n^{\circ} 12$ de 02 de janeiro de 2001, dispõe como critérios de qualidade o valor máximo de $10^{2} \mathrm{NMP} / \mathrm{g}$ para Coliformes á $45^{\circ} \mathrm{C}$ e ausência de Salmonella spp. em $25 \mathrm{~g}$, para que este alimentos sejam considerados adequados ao consumo humano, disposto no item 1 (c) do Anexo 1 dessa normatização (BRASIL, 2001).

No Brasil, a incidência de doenças infecciosas e parasitárias ainda é expressiva em determinadas regiões, onde o acesso ao saneamento e serviços de saúde ainda é deficitário. A bactéria entérica Salmonella spp. é uma das principais causadoras dos casos de infecção alimentar no país, cujo conjunto de sinais e sintomas incluem dores abdominais, diarreias, náuseas, febre e falta de apetite (SHINOHARA et al., 2008).

A Salmonella spp. está amplamente distribuída no ambiente, sendo encontrada no solo, água, estando ainda disseminadas em animais, seres humanos e até mesmo em equipamentos. As aves e bovinos são um dos maiores contribuintes para disseminação deste patógeno (BRASIL, 2008).

Atualmente, Coliformes vem ocasionando surtos epidêmicos, principalmente em indivíduos com competência imunológica comprometida. O patógeno está presente em solos e vegetais, com fácil capacidade de replicação e pode ser encontrado em ambientes externo e na microbiota intestinal de animais de sangue quente (DIAS et al., 2010). A presença de Coliformes em alimentos deve ser levada em consideração, visto que esta pode ser interpretada como má condição higiênica, sinalizando a necessidade de um controle mais efetivo e contínuo na linha de produção, por exemplo (PORTO et al., 2011).

Considerando a segurança higiênico-sanitária dos produtos comercializados no município de Belém do Pará, o presente estudo objetivou realizar análise microbiológica de polpas industrializadas vendidas em supermercados de Belém do Pará.

\section{DESENVOLVIMENTO: MATERIAIS E MÉTODOS}

\subsection{Amostras}

As 30 amostras de polpas de frutas, incluindo os sabores de cupuaçu, graviola, manga, goiaba, acerola, maracujá, caju, muruci, abacaxi, açaí e cacau foram adquiridas em um supermercado da cidade de Belém do Pará, no período compreendido de fevereiro de 2013 a março de 2014, e conduzidas até o laboratório de higiene de alimentos do Centro Universitário do Estado do Pará-CESUPA para realização das análises microbiológicas de Salmonella spp. e Coliformes á $45^{\circ} \mathrm{C}$. 


\subsection{Análises microbiológicas}

A caracterização dos micro-organismos a serem pesquisados nas análises microbiológicas esteve embasada nos padrões preconizados pela legislação em vigor RDC № 12 de 2 de janeiro de 2001.

Para detecção Salmonella spp., conforme Figura 1 (SILVA; JUNQUEIRA; SILVEIRA, 2001), pesou-se $25 \mathrm{~g}$ da amostra, que foi transferida para um frasco de 225 $\mathrm{ml}$ água tamponada, sendo diluída e incubada a $35^{\circ} \mathrm{C}$ por 24 horas, caracterizando 0 pré-enriquecimento em caldo seletivo que tem como objetivo recuperar as células injuriadas, restabelecendo as condições fisiológicas ideais para crescimento e multiplicação dos micro-organismos. Após o pré-enriquecimento transferiu-se, com auxílio de uma pipeta, o volume de $1 \mathrm{ml}$ do cultivo para o tubo contendo caldo Tetrationato (TT) e para o tubo com caldo Selenito Cistina (SC), sendo incubados à $35^{\circ} \mathrm{C}$ por 24 horas. Essa etapa é o enriquecimento em caldo seletivo, que visa inibir a multiplicação da microbiota acompanhante e promover a elevação preferencial do número de células de Salmonella spp.

Após o período de incubação, iniciou-se o plaqueamento diferencial, ocorrendo a agitação dos tubos de TT e SC, retirando-se uma alçada dos referidos caldos para as placas de Ágar Entérico Hectoen (HE), Ágar Bismuto Sulfito (BS), Ágar Xilose Lisina Desoxiciolato (XLD), Ágar Salmonella-Shigella (SS), Ágar Verde Brilhante (VB) incubando as placas de forma invertidas a $35{ }^{\circ} \mathrm{C}$ por 24 horas e verificando-se 0 desenvolvimento de colônias típicas para Salmonella spp. Em caso positivo, com auxílio de uma agulha de inoculação, removeu-se do centro da colônia um porção da massa de células, que fora inoculada em tubos de Ágar Tríplice Açúcar Ferro (TSI), Ágar Lisina Ferro (LIA), Caldo Malonato (MAL) e teste de Urease (UREIA), respectivamente. Esta etapa teve a finalidade de verificar as características bioquímicas diferenciais da Salmonella spp. (SILVA; JUNQUEIRA; SILVEIRA, 2001). 
Figura 1 - Esquema para análise de Salmonella spp.

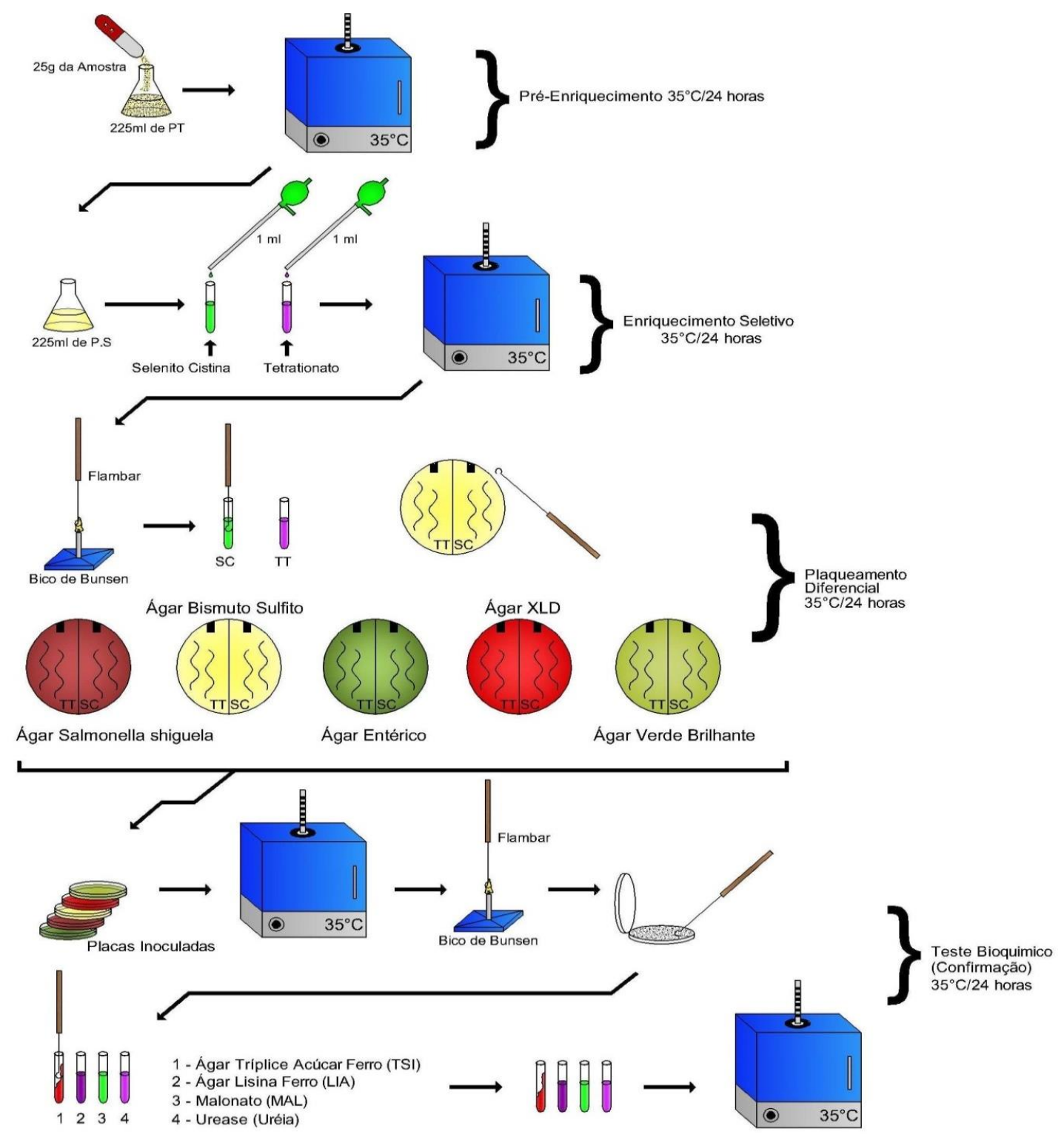

Fonte: Adaptado de Silva, Junqueira e Silveira, 2001.

Para a contagem de Coliformes a $45^{\circ} \mathrm{C}$, conforme figura 2 (SILVA; JUNQUEIRA; SILVEIRA, 2001), pesou-se de $25 \mathrm{~g}$ da amostra homogeneizada, transferindo-a para um frasco contendo $225 \mathrm{ml}$ de água peptonada simples, os quais foram diluídos dando origem à diluição $10^{-1}$. Após este procedimento foi transferido, com o auxílio de uma pipeta, $1 \mathrm{ml}$ dessa diluição para um tubo de ensaio contendo $9 \mathrm{ml}$ de água peptonada simples, originando a diluição $10^{-2}$. Desta diluição foi retirado $1 \mathrm{ml}$ com auxílio de uma pipeta, sendo transferido, em seguida, para um outro tubo de ensaio contendo $9 \mathrm{ml}$ de água peptonada, dando origem à diluição $10^{-3}$.

Realizou-se o teste presuntivo com uma série de três tubos de ensaio para cada diluição, contendo $9 \mathrm{ml}$ de Caldo Lactosado (CL) e um tubo de Durham invertido. 
Transferiu-se $1 \mathrm{ml}$ das diluições $10^{-1}, 10^{-2}$ e $10^{-3}$ para cada tubo da série de três. Esses tubos foram incubados a $35^{\circ} \mathrm{C}$ por 48 horas. Nos tubos positivos, com produção de gás no interior dos tubos de Durhan, prosseguiu-se com a análise, retirando uma alçada desse cultivo, que foi inoculada em tubos contendo Caldo de E.coli (EC), incubados em estufa a $45^{\circ} \mathrm{C}$ por 24 horas em banho maria, caracterizando o teste confirmativo para Coliformes termotolerantes. O teste, assim como o anterior, considerou positivo os tubos em que houve produção de gás no interior do tubo de Durhan. Após determinação da trinca microbiológica foi determinado o número mais provável (NMP), baseado em tabela proposta por Silva, Junqueira e Silveira (2001).

Figura 2 - Esquema para análise de Coliformes à $45^{\circ} \mathrm{C}$.
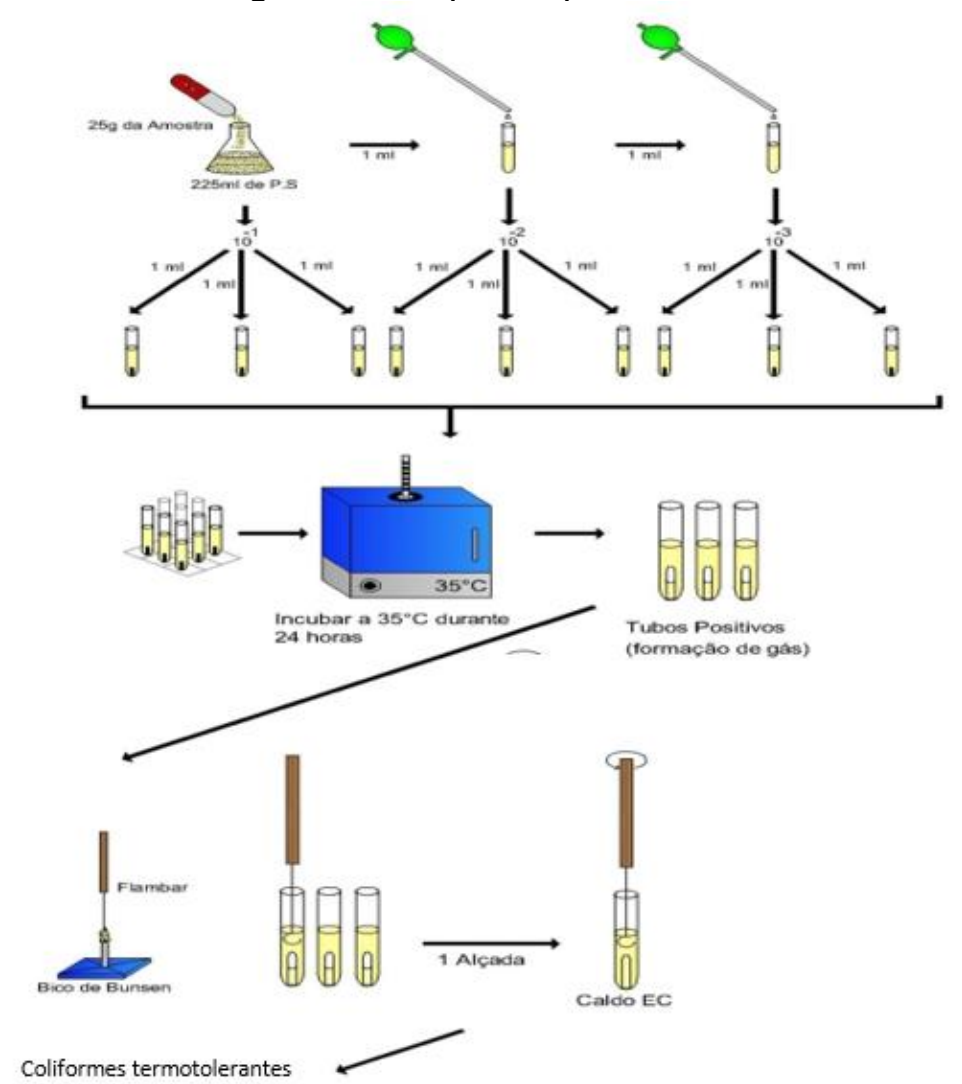

Coliformes termotolerantes

Teste Positivo Teste Negativo
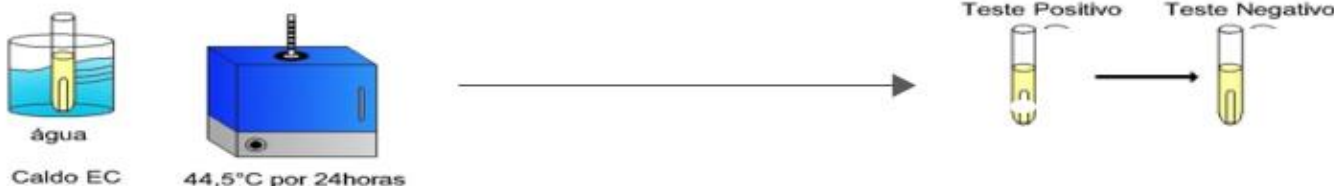

Fonte: Adaptado: Silva, Junqueira e Silveira, 2001.

A tabulação dos dados foi realizada utilizando Microsoft Excel versão 2010.

\section{RESULTADOS E DISCUSSÃO}

Segundo o Ministério da Agricultura Pecuária e Desenvolvimento (MAPA), a polpa de fruta é definida "como o produto não fermentado, não concentrado, não diluído, 
obtido de frutos polposos, por meio de processo tecnológicos adequados, com teor mínimo de sólidos totais, provenientes da parte comestível do fruto" (BRASIL, 2000). A polpas podem ser produzidas a partir de uma única espécie ou de duas ou mais espécies de frutas, podendo ser classificadas como simples ou mista, originárias de frutas saudáveis e isentas de contaminações microbiológicas, não podendo apresentar fragmentos de partes não-comestíveis da fruta, nem serem acrescidas substâncias que não façam parte de sua composição natural (EMBRAPA, 2005).

O processamento de polpa de frutas congeladas deve obedecer aos padrões preconizados pelo Ministério da Agricultura, Pecuária e Abastecimento e pela Agência Nacional de Vigilância Sanitária (Anvisa), sendo essencial o cumprimento destas medidas para que os alimentos sejam produzidos com qualidade e segurança (EMBRAPA, 2005).

Os resultados obtidos após as análises de Coliformes a 45ำ e Salmonella spp. das 30 amostras das polpas congeladas de frutas estão expostos na Tabela 1.

Tabela 1 - Análise microbiológicas das polpas de frutas comercializadas em supermercados de Belém do Pará (continuação).

\begin{tabular}{|c|c|c|}
\hline Polpa & Coliformes á $45^{\circ} \mathrm{C}$ & Salmonella spp. (NMP/g) \\
\hline 1. Cupuaçu & $<11 \mathrm{NMP} / \mathrm{g}$ & Ausência em 25g \\
\hline 2. Cupuaçu & $<3 N M P / g$ & Ausência em 25g \\
\hline 3. Cupuaçu & $<3 N M P / g$ & Ausência em 25g \\
\hline 4. Cupuaçu & $<3 N M P / g$ & Ausência em 25g \\
\hline 5. Cupuaçu & $<3 \mathrm{NMP} / \mathrm{g}$ & Ausência em 25g \\
\hline 6. Manga & $<11 \mathrm{NMP} / \mathrm{g}$ & Ausência em 25g \\
\hline 7. Manga & $<3 N M P / g$ & Ausência em 25g \\
\hline 8. Goiaba & $<3 N M P / g$ & Ausência em $25 \mathrm{~g}$ \\
\hline 9. Goiaba & $<3 N M P / g$ & Ausência em 25g \\
\hline 10. Goiaba & $<3 N M P / g$ & Ausência em 25g \\
\hline 11. Acerola & $<3 N M P / g$ & Ausência em 25g \\
\hline 12. Acerola & $<3 N M P / g$ & Ausência em 25g \\
\hline 13. Acerola & $<3 N M P / g$ & Ausência em 25g \\
\hline 14. Acerola & $<3 N M P / g$ & Ausência em 25g \\
\hline 15. Acerola & $<3 N M P / g$ & Ausência em $25 \mathrm{~g}$ \\
\hline 16. Maracujá & $<3 N M P / g$ & Ausência em 25g \\
\hline 17. Maracujá & $<3 N M P / g$ & Ausência em $25 \mathrm{~g}$ \\
\hline 18. Maracujá & $<3 N M P / g$ & Ausência em 25g \\
\hline 19. Caju & $<3 N M P / g$ & Ausência em 25g \\
\hline 20. Caju & $<3 N M P / g$ & Ausência em 25g \\
\hline 21. Caju & $<3 N M P / g$ & Ausência em 25g \\
\hline 22. Muruci & $<3 N M P / g$ & Ausência em 25g \\
\hline 23. Abacaxi & $<3 \mathrm{NMP} / \mathrm{g}$ & Ausência em 25g \\
\hline
\end{tabular}


Tabela 1. Análise microbiológicas das polpas de frutas comercializadas em supermercados de Belém do Pará
24. Abacaxi
25. Abacaxi
$<3 N M P / g$
26. Açaí
$<3 N M P / g$
27. Açaí
$240 \mathrm{NMP} / \mathrm{g}$
28. Cacau
$>1.100 \mathrm{NMP} / \mathrm{g}$
29. Graviola
$<3 \mathrm{NMP} / \mathrm{g}$
Ausência em $25 \mathrm{~g}$
Ausência em 25g
30. Graviola
$<3 N M P / g$
Ausência em $25 \mathrm{~g}$
Ausência em $25 \mathrm{~g}$
Ausência em $25 \mathrm{~g}$
Ausência em $25 \mathrm{~g}$
$<3 \mathrm{NMP} / \mathrm{g}$
Ausência em $25 \mathrm{~g}$

(conclusão).

Padrão *

$10^{2} \mathrm{NMP} / \mathrm{g}$ *

Ausência em 25g*

${ }^{*}$ RDC no12 de janeiro de 2001

Fonte: Pesquisa de campo (2014).

A RDC №12 de janeiro de 2001, que dispõe sobre o padrão microbiológico para alimentos no Brasil, estabelece como máximo tolerável de Coliformes a $45^{\circ} \mathrm{C}$, em amostra indicativa, de polpas de frutas $10^{2} \mathrm{NMP} / \mathrm{g}$, conforme o item 1c do Anexo 1 dessa resolução. Os resultados nas análises do presente estudo (Tabela 1) demonstraram que apenas duas amostras analisadas apresentaram contagens acima dos padrões preconizados pela legislação em vigor, com valor >1100 NMP/g e de $240 \mathrm{NMP} / \mathrm{g}$, correspondendo a $6,66 \%$ do total de amostras. Ao se analisar os resultados por grupo de fruta, nota-se inadequação em $100 \%$ das amostras de açaí, com contagem acima do preconizado pela legislação. Este fato ocorreu devido um conjunto de fatores que incluem uma elevada carga microbiana, falhas no processamento e/ou armazenamento, além de interferentes como temperatura e umidade relativa. Estudos recentes estão demonstrando elevada prevalência de contaminação de polpas congeladas e sucos in natura comercializados no país, como descrito por Faria, Oliveira e Costa (2012) que identificou a presença de Coliformes termotolerantes em cinco das 36 amostras analisadas, totalizando $13,8 \%$.

O índice referente a contagem de Coliformes a $45^{\circ} \mathrm{C}$ é utilizado para avaliar as condições higiênicas de toda cadeia produtiva que envolve desde a produção até o processamento e estocagem, sendo necessário seguir diretrizes de boas práticas de produção e comercialização de produtos. A presença deste grupo de micro-organismos) sugere contaminação por Escherichia coli, que possui como habitat o intestino humano e de outros animais endotérmicos (SOUZA; CARNEIRO; GONSALVES, 2011).

Em estudo de Dantas et al., (2012) a análise de 19 amostras de diferentes sabores de polpa de frutas comercializadas na cidade de Campina Grande (PB) apresentou $100 \%$ de resultado coerente em relação aos Coliformes à $45^{\circ} \mathrm{C}$, sendo que a mesma adequação à legislação em vigor foi também identificada nos trabalhos de Feitosa et al, (1997) e Freire et al. (2009). 
Por se tratar de um micro-organismo com elevado grau de patogenicidade, a Salmonella spp. de acordo com a RDC № 12/01, deve estar ausente em $25 \mathrm{~g}$ da amostra analisada, os resultados expostos na tabela 1, demonstraram que $100 \%$ das 30 amostras analisadas de polpas de frutas com diferentes sabores, apresentaram-se adequadas segundo o padrão citado.

Batista et al. (2013), em pesquisa realizada com polpas congeladas selecionadas aleatoriamente, selecionadas aleatoriamente e comercializadas em uma agroindústria localizada no município de Datas - MG, detectou ausência de Salmonella spp. nas amostras avaliadas. Resultados semelhantes, com adequação aos padrões da legislação vigente, também foram obtidos em análises qualitativas efetuadas no período de março de 2007, em amostras comerciais processadas e distribuídas por redes de supermercados e restaurantes no estado de São Paulo (FREIRE et al, 2009).

Contudo, Dantas et al., (2012) que realizaram um levantamento da qualidade microbiológica de quatro marcas de polpas de frutas em Campina Grande-PB detectaram presença de Salmonella spp. em três das marcas selecionadas, indicando assim que estas indústrias comercializam polpas de frutas fora dos padrões legais.

A presença de Salmonella spp. em alimentos é um grave problema de saúde pública e é indicativo de condições higiênico sanitárias insatisfatórias e/ou irregulares, pois as medidas de prevenção, como a adoção de boas práticas de fabricação e comercialização não foram executadas, culminando, então, em risco direto para o consumidor (SHINOHARA et al., 2008).

\section{CONCLUSÃO}

Os resultados das análises microbiológicas obtidos por meio deste estudo, apresentaram-se de acordo com os limites preconizados pela legislação vigente, RDC $\mathrm{n}^{\circ} 12$ de 02 de janeiro de 2001, quanto a Salmonella spp. no qual o grupo amostral apresentou ausência do microrganismo em $25 \mathrm{~g}$ em todas as amostras analisadas, porém duas das análises realizadas em polpas congeladas de açaí, indicaram possíveis irregularidades quanto aos aspectos higiênicos referente a Coliformes a $45^{\circ} \mathrm{C}$, com $240 \mathrm{NMP} / \mathrm{g}$ e $>1100 \mathrm{NNMP} / \mathrm{g}$, valores estes encontram-se acima do padrão de $10^{2} \mathrm{NMP} / \mathrm{g}$ estabelecido pela RDC n¹2/2001.

Assim, ressalta-se a relevância da avaliação da qualidade microbiológica das polpas de frutas comercializadas para saúde dos consumidores, bem como a necessidade de aplicação de medidas preventivas e/ou corretivas em todas as etapas da cadeia produtiva.

\section{REFERÊNCIAS}


AQUINO, R. C.; PHILIPPI, S. T. Consumo infantil de alimentos industrializados e renda familiar na cidade de São Paulo. Rev Saúde Pública, v. 36, n. 6, p. 655-60, 2002.

BALBANI, A.P.S.; BUTUGAN, O. Contaminação biológica de alimentos. Revista de Pediatria, v. 23, n. 4, 2001.

BATISTA, A. G. et al. Parâmetros de qualidade de polpas de frutas congeladas: uma abordagem para produção do agronegócio familiar no Alto Vale do Jequitinhonha. Tecnologia e Ciência Agropecuária, v.7, n. 4, p. 49-54, 2013.

BRASIL. MINISTÉRIO DA AGRICULTURA DO ABASTECIMENTO. Instrução Normativa $n^{\circ}$ 01/00, de 07/01/00. Regulamento técnico geral para fixação dos padrões de identidade e qualidade para polpa de fruta. Diário Oficial da União, Brasília, DF, 2000.

BRASIL. Ministério da Saúde. Análise Epidemiológica dos Surtos de Doenças Transmitidas por Alimentos no Brasil. Brasília, 2008. Disponível em:

http://portal.saude.gov.br/portal/arquivos/pdf/surtos_dta.pdf. Acesso em: 03 abr. 2014.

BRASIL. RDC n`12 de 02 de janeiro de 2001. 2001.Disponível em:

http://portal.anvisa.gov.br/documents/33880/2568070/RDC_12_2001.pdf/15ffddf63767-4527-bfac-740a0400829b. Acesso em: 03 set. 2017.

CRUZ, F. T.; SCHNEIDER, S. Qualidade dos alimentos, escalas de produção e valorização de produtos tradicionais. Revista Brasileira de Agroecologia, v. 5, n. 2, p. 22-38, 2010.

DANTAS, R. L. et al. Qualidade microbiológica de polpa de frutas comercializadas na cidade de Campina Grande, PB. Revista Brasileira de Produtos Agroindustriais, v. 14, n. 2, p. 125-130, 2012.

DIAS, M. T. et al. Avaliação da sensibilidade de cepas de Escherichia coli isoladas de mexilhões (Perna perna linnaeus, 1758) à antimicrobianos. Ciênc. Tecnol. Aliment., v. 30, n. 2, 2010.

EMBRAPA. Polpa de fruta congelada. Agroindústria familiar. Brasília, DF. 2005.

FARIA, M.; OLIVEIRA, L. B. D.; COSTA, F. E. C. Determinação da qualidade microbiológica de polpas de açaí congeladas comercializadas na cidade de Pouso Alegre - MG. Alim Nutr., v. 23, n. 2, 2012.

FEITOSA, T. et al. Perfil microbiológico de polpa de frutas produzidas e comercializadas nos estados do Ceará e Rio Grande do Norte. B. CEPPA, v. 15, n. 1, p. 65-74,1997.

FREIRE. M. T. A. et al. Caracterização físico-química, microbiológica e sensorial de polpa de cupuaçu congelada (Theobroma grandiflorum Schum). Braz. J. Food Technol., v. 12, n. 1, p. 09-16, 2009.

HARVEY, M.; MCMEEKIN, A.; WARDE, A. Qualities of food. New York: Palgrave, 2004.

MESQUITA, M. O. et al. Qualidade microbiológica no processamento do frango assado em unidade de alimentação e nutrição. Ciência Tecnologia de Alimentos, v. 26, n. 1, p.198-203, 2006. 
PARIZ, K. L. Avaliação da qualidade microbiológica de polpas de frutas. Trabalho de Conclusão de Curso (graduação) - Instituto Federal de Educação, Ciência e Tecnologia do Rio Grande do Sul, Bento Gonçalves, 2011.

PORTO, M. A. L. et al. Coliformes em água de abastecimento de lojas fast-food da Região Metropolitana de Recife (PE, Brasil). Ciênc. saúde coletiva, v.16, n.5, 2011.

SANTOS, M.; TONDO, E. C. Determinação de perigos e pontos críticos de controle para implantação de sistema de análise de perigos e pontos críticos de controle em lactário. Revista Nutrição, v.13, n. 3, p.211-222, 2000.

SILVA, N. da; JUNQUEIRA, V. C. A.; SILVEIRA, N. F. de A. Manual de Métodos de Análise Microbiológica de Alimentos. 2. ed. São Paulo: Varela, 2001.

SIQUEIRA. L. P. et al. Avaliação microbiológica da água de consumo empregada em unidades de alimentação. Ciênc. saúde coletiva, v.15, n.1, p. 63-66, 2010.

SHINOHARA, N. K. S. et al. Salmonella spp., importante agente patogênico veiculado em alimentos. Ciênc. saúde coletiva, v. 13, n. 5, 2008.

SOUZA, G. C.; CARNEIRO, J. G.; GONSALVES, H. R. O. Qualidade microbiológica de polpas de frutas congeladas produzidas no município de Russas - CE. ACSA -

Agropecuária Científica no Semi-Árido, v. 7, n. 3, 2011. 